\title{
La concepción constructivista como modelo explicativo del aprendizaje en los deportes de equipo"
}

The constructivist approach as an explanatory model in team sports

Recibido: noviembre 23 de 2009 | Revisado: mayo 6 de 2010 | Aceptado: julio 15 de 2010

\author{
Francisco Alarcón LÓPEZ ** \\ Universidad Católica San Antonio de Murcia, España \\ DAVID CÁRDENAS VÉLEZ ${ }^{* * *}$ \\ MARÍA IsABEL PiÑAR LÓPEZ \\ María TERESA MIRANDA LEÓN ${ }^{* * * * *}$ \\ Universidad de Granada, España \\ NuRIA UREÑA ORTÍN \\ Universidad de Murcia, España
}

\section{RES UMEN}

El objetivo de este trabajo es exponer cómo se hace necesario utilizar la teoría constructivista, para poder entender los mecanismos que se producen en el aprendizaje de los deportes de equipo. Éstos poseen unas características propias que dan lugar a numerosas habilidades, las cuales requieren, además de un dominio motriz, de la capacidad, por parte del jugador, de saber cuándo y dónde utilizarlas, es decir, se necesita un proceso de comprensión de la lógica del juego para poder jugar. La mayoría de los modelos de enseñanza que se utilizan en la actualidad dentro del entrenamiento deportivo, todavía se basan en teorías de aprendizaje asociativas, en las que el jugador debe adquirir una serie de habilidades de manera descontextualizada, siendo esta enseñanza insuficiente para el entendimiento de los deportes de equipo.

Palabras clave autores

Constructivismo, aprendizaje, deportes de equipo.

Palabras clave descriptores

Psicología del desarrollo, psicología de la educación, proceso de aprendizaje, psicofisiología, desarrollo motor, educación física.

\section{A B S T R A C T}

The aim of this paper is to explain how it is necessary to use constructivist theory to understand the mechanisms that occur in the learning of team sports. These have distinct characteristics that give rise to many skills, which also require a motor domain, the capacity of each player to know when and where to use them, i.e. it needs a process of understanding the logic of the game to play. Most of the teaching models that are currently used in sports training is still based on theories of associative learning in which the player must acquire a skill set so decontextualized, this teaching being insufficient for understanding the team sports.

Key words authors

Constructivism, learning, team sports.

Key words plus

Developmental Psychology, Educational Psychology, Learning Processes,

Psychophysiology, Motor Development, Physical Education. 
Las condiciones de compañeros y adversarios en espacios reducidos y el poco tiempo que existe durante las acciones colectivas en los deportes de equipo, hace que existan unas condiciones muy altas de incertidumbre, que hacen que tomar decisiones sea de una gran dificultad. Poseer un gran conocimiento sobre el juego va a ayudar a prever cuál de las posibles alternativas es la más adecuada según la experiencia adquirida, y así poder disminuir el grado de incertidumbre (Alarcón, 2008).

Anderson (1987) propuso dos tipos de conocimiento: declarativo y procedimental. El conocimiento declarativo es el conjunto de atributos y características que se dice de un objeto, un suceso o una idea. Es el concepto que se tiene de algo. Se identifica como "el saber", "saber decir", "saber qué". Este tipo de conocimiento hace referencia al conocimiento que tienen los deportistas de las habilidades y estrategias de juego. Su característica principal es que puede ser verbalizado, ser declarado, de ahí su nombre. El conocimiento procedimental se refiere a cómo realizar las diferentes acciones deportivas, a las reglas que se aplican para solventar los diferentes problemas que surgen en las situaciones deportivas (Ruiz \& Arruza, 2005), es decir, "saber cómo". Para Coll y Valls (1992) un procedimiento "es un conjunto de acciones ordenadas, orientadas a la consecución de una meta" (p. 84).

Varios autores han sugerido que una base de conocimiento declarativo es necesaria para el desarrollo de estructuras más complejas de conocimiento procedimental (Anderson, 1976, 1982; Chi $\&$ Rees, 1983). En este sentido, French y Thomas (1987) señalan que uno debe primero desarrollar una base de conocimiento declarativo dentro de un deporte dado, antes de poder desarrollar adecuadamente buenas destrezas en la toma de decisiones.

Ahora bien, icómo adquiere el jugador el conocimiento declarativo necesario para ser eficiente en los deportes de equipo? Cada una de las teorías del aprendizaje intenta dar respuesta a cómo aprende el sujeto (Santiuste-Bermejo \& SantiusteDíaz, 2008). Las correspondientes al paradigma cognitivo que, según autores como Coll y Valls (1992) o Pozo (2006) son las que mejor explican el aprendizaje del conocimiento necesario para la práctica de los deportes de equipo, se pueden dividir en aquellas que entienden la construcción del conocimiento de una manera estática y las que lo entienden de una manera dinámica. Por las características del propio deporte, será necesario utilizar ambas vertientes. La primera, compatible con las leyes asociativas del aprendizaje, explica los mecanismos de automatización de habilidades deportivas necesarios para liberar la capacidad de atención; mientras la segunda, que es en la que se basa las teorías del constructivismo, es necesaria para entender los mecanismos que se producen cuando el jugador adquiere un conocimiento complejo basado en la interconexión de un grupo de conceptos. Esto es imprescindible en los deportes de equipo, puesto que la consecución del éxito pasa por ser capaz de saber qué y cuándo utilizar las diferentes habilidades según las demandas del entorno (adversarios, compañeros, espacios libres, etc). En ambas, el aprendizaje parte de los conocimientos previos del aprendiz, pero desde el primer enfoque la nueva información se asimila a las estructuras de conocimiento ya existentes sin modificarlas, de manera estática. En cambio, desde la segunda perspectiva, estos conocimientos previos van a ser modificados por la nueva información, es decir, existe un proceso de reestructuración de los conocimientos anteriores, más que una sustitución de unos conocimientos por otros (Pozo, 2006).

Hay que tener claro que tanto las teorías asociativas como las cognitivas tienen justificación cuando se pretenden explicar los procesos de aprendizaje del ser humano. Según Beltrán, García-Alcaniz, Moraleda, Calleja y Santiuste (1992), estas posiciones no son contradictorias sino complementarias, y cada una tendrá mayor relevancia explicativa según el nivel en el que ocurra el aprendizaje, aunque, según los autores:

Los psicólogos siguen resistiéndose a adoptar una visión unitaria del aprendizaje y a admitir la existencia de un único proceso que gobierne todas las formas de aprendizaje posibles, aceptando, por el contrario, la existencia de teorías explicativas distintas para explicar procesos también distintos. (p. 58) 
Como señalan Carretero (1985) y Coll (1996), existe un combate inútil entre enfoques decididos a no entenderse, ya que hablan de cosas diferentes y manejan bases de datos distintas.

Según Coll (1991), los referentes teóricos, desde el paradigma cognitivo (en sus dos vertientes) y del ecológico, sirven de base para los conceptos y principios que utiliza la concepción constructivista de la educación, que prestan atención a determinados aspectos o factores del desarrollo y del aprendizaje, y que coinciden todos ellos en señalar que el desarrollo y el aprendizaje humano son el resultado de un proceso de construcción.

Así pues, el proceso de aprendizaje se puede analizar de diferentes niveles, no teniendo que ser excluyentes. La mente humana sería un sistema complejo (Aparicio-Serrano \& Hoyos, 2008; García, 1995) que podría ser analizado desde distintos niveles o planos de complejidad, que reciben una explicación adecuada desde cada paradigma. Según Pozo (2006), se pueden identificar cuatro niveles distintos de complejidad. Para el análisis sobre la adquisición del conocimiento necesario para deportes de equipo, se va a partir de los mismos planos de complejidad que propone este autor: el segundo y tercer nivel de análisis, es decir, el proceso de adquisición y cambio de las representaciones, y la función de la conciencia reflexiva. El autor descarta el primer nivel, no porque no sea un modelo explicativo válido, ya que estos dos niveles anteriores se pueden explicar desde este primero, sino porque cree que las teorías conexionistas

(...) están lejos de hacer aportaciones sustanciales en aprendizajes más complejos, y difícilmente puede servir como guía principal para que aprendices y maestros comprendan mejor los procesos de aprendizaje en contextos reales y logren hacerlos más efectivos, (....), y éstos apenas permiten vislumbrar soluciones a los problemas de aprendizaje que nos aquejan. (pp. 108-109)

Del cuarto plano de análisis, Pozo (2006) cree, al igual que Coll (2004), que toda representación se construye en la interacción social y solo puede entenderse si se analiza como una construcción so- cial, pero concibe el aprendizaje como un proceso interno al organismo, por lo que, por más que esté motivado en la interacción social, finalmente, las representaciones tienen su sede en la mente individual (Rodrigo, 1994), y cambian por procesos cognitivos propios de la mente.

Establecido el punto de partida, cada tipo de conocimiento necesita de un tipo de aprendizaje diferente; conocido ya lo primero, lo que hay que saber es qué tipos de aprendizaje existen. Para ello, utilizaremos el baloncesto como ejemplo de deporte de equipo.

Uno de los problemas por el cual las teorías parecen que no son compatibles, es que sus puntos de partida son diferentes, tomando cada una de ellas una sola perspectiva. El aprendizaje se puede analizar partiendo desde diferentes criterios y para saber qué tipos de aprendizajes existen habrá que conocer cuáles son cada uno de los primeros. Para conocer estos criterios, se partirá de las teorías explicativas de la conducta humana.

Cuando un sujeto percibe información del exterior, esta es procesada, comparándola con el conocimiento que ya se posee en la memoria, para programar la respuesta más adecuada. Desde esta perspectiva del procesamiento de información de la mente humana, existirían dos sistemas de memoria con características y funciones diferentes: la memoria de trabajo y la memoria permanente. Estas dos memorias tienen una gran importancia en los procesos de aprendizaje, tanto en la adquisición como en la recuperación. Dependiendo de cómo se procesa y se almacena esa información y cómo se recupera, existirán un tipo u otro de aprendizaje. Por lo tanto, los criterios de clasificación de los tipos de aprendizajes serán:

\section{Atendiendo al proceso de adquisición}

\section{- Según el grado de consciencia}

Según Pozo (2006), al ser la atención un mecanismo limitado, no puede obtener toda la información del exterior. Así, cuando un sujeto no es capaz de atender a aspectos del entorno, no puede ser consciente de ellos, aunque en condiciones muy 
determinadas y favorables se puede materializar el aprendizaje (Reber, 1993); es lo que se ha llamado el aprendizaje de teorías implícitas (Aparicio-Serrano \& Hoyos, 2008; Núñez, 1998; Pozo, 2002, 2006). Es posible que estas teorías que se adquieren de manera inconsciente, no correspondan siempre con una interpretación adecuada de la realidad. En el caso del baloncesto como ejemplo de un deporte de equipo, un jugador puede haber adquirido un concepto de cómo utilizar un bloqueo directo (BD) de manera inconsciente, basado en su experiencia, que le ha dictado que el objetivo de jugar el $\mathrm{BD}$ es dirigirse hacia el aro. Este aprendizaje se almacena en la memoria permanente de una manera muy determinada, como se verá a continuación.

\section{- Según las operaciones realizadas en la memoria de trabajo}

La capacidad limitada de la memoria de trabajo restringe la información que puede ser atendida. Una operación que incrementa esta capacidad es condensar elementos de información que tienden a producirse juntos en forma de piezas de información, que se almacenan y recuperan como una única representación. Es lo que ocurre cuando se aprende un número de teléfono, el sujeto condensa o agrupa en trozos el teléfono en vez de memorizar cifra a cifra (Pozo, 2006). Para la memoria de trabajo, lo realmente limitante no es la cantidad de información, sino el número de elementos independientes que deben procesarse. Cuando varios elementos se condensan en uno solo consumen los recursos atencionales propios de un elemento.

Pero no sólo se condensa información verbal. También los procedimientos pueden pasar por esta operación de agrupación. Esta es una de las explicaciones que se dan para entender las diferencias entre expertos y noveles en tareas motrices. Así Anderson (1983) explica en su teoría cómo se adquiere conocimiento procedimental mediante estas operaciones, que él llama proceso de compilación. Según este autor, el conocimiento declarativo es transferido al conocimiento procedimental a través de estos procesos de compilación de conocimiento.
La condensación de información ya sea verbal o procedimental, no está relacionada directamente con ninguna forma de almacenar esta información en la memoria permanente, es decir, dependiendo de las estrategias de instrucción utilizadas para su aprendizaje, podrá organizarse de una manera más asociativa o más comprensiva, como se analizará en el punto siguiente. Como dice Pozo (2006), a medida que el aprendizaje se acompañe de una comprensión de los mecanismos que subyacen a esos agrupamientos, basada en un aprendizaje constructivo, mayor sentido adquirirá esa condensación. Así, por ejemplo, cuando un jugador condensa una jugada, "no sólo sabe reconocerla rápidamente sino que también comprende cuándo, cómo y contra quién hay que utilizarla" (p. 149).

\section{- Según se almacene en la memoria permanente}

Una vez procesada la nueva información y comparada con el conocimiento previo, ésta se almacenará en la memoria permanente. Este tránsito de la memoria de trabajo a la memoria permanente está medido por un conjunto de procesos de adquisición, y no solo dependerá de los recursos cognitivos que se hayan utilizado en la memoria de trabajo, sino sobre todo en la forma más o menos organizada en que se traslade a la memoria permanente (Pozo, 2006). Existen dos factores que influyen en la organización de esta información, según cómo sea la representación que se forme del nuevo conocimiento, y según cómo esté relacionada esta representación mental con el resto de representaciones que se poseen.

\section{- Según el tipo de representación}

Desde la psicología cognitiva se ha hecho un gran esfuerzo, durante décadas, por entender cómo se representa el conocimiento sobre el mundo, existiendo algunas teorías que explican la formación de esquemas más o menos generales, y otras, éstas más modernas, que intentan explicar la formación de modelos mentales. 
Cuando un jugador es expuesto a una situación de juego, realiza una representación singular del suceso. Estas representaciones son llamadas modelos mentales, siendo definidas como representaciones episódicas que incluyen personas, objetos y sucesos enmarcados en unos parámetros espaciales, temporales, intencionales y causales (De Vega, Díaz \& León, 1999). Es lo que le ocurre a un jugador que memoriza la realización de un medio colectivo como el bloqueo indirecto (BI) en baloncesto, en el que éste pasa por un proceso de condensación de información para atender a todos los estímulos presentes, incluidos los afectivos o emocionales, como la sensación de satisfacción cuando se produce el éxito.

Un ejemplo de modelo mental es el conocimiento que se adquiere de hechos o datos (Pozo, 1992). Éstos deben aprenderse literalmente, de un modo reproductivo, como un número de teléfono. Para esto, el sujeto debe hacer una copia más o menos literal o exacta de la información proporcionada y almacenarla en su memoria. Para que no desaparezca de la memoria, los hechos deben ser repasados o practicados cada cierto tiempo. Esta nueva práctica no añade nada nuevo al aprendizaje, sólo evita el olvido. Por lo tanto, según el autor, se está utilizando un tipo de aprendizaje memorístico. Es lo que sucedió en el caso anterior del jugador que retiene un modelo episódico de un BI.

Estas pautas de activación de redes de unidades simbólicas que se almacenan en la memoria, pueden ser más o menos densas. En el caso anterior, en el modelo mental de BI se podía haber almacenado también relaciones con otros hechos y sucesos del contexto. Es decir, los jugadores pueden establecer relaciones entre una determinada actuación suya y la conducta de aceptación del entrenador. Esto puede generar un reforzamiento positivo de manera inconsciente; es lo que se ha denominado un aprendizaje asociativo (Pozo, 1989). Según Beltrán et al. (1992), en el entrenamiento, es muy frecuente este aprendizaje respondiente o condicionamiento, sobre todo de carácter emocional o afectivo.

Este aprendizaje puede ser también consciente y se puede memorizar un número mayor de relacio- nes dentro de la representación mental. Es lo que sucede cuando existe un entrenamiento específico de un contenido de juego como el $\mathrm{BD}$, en el que se le enseña al jugador que cuando ocurra una serie de circunstancias de juego, debe actuar de una determinada manera, por ejemplo, cuando el adversario del jugador con balón del BD se coloque detrás de éste para impedir ser bloqueado, debería progresar hacia el aro botando, siendo esto reforzado positivamente por el entrenador. Cuando la situación del BD se repite en situaciones idénticas o parecidas, puede acabar por almacenarse como un esquema estable y genérico. Es lo que ocurre cuando se repite en la vida cotidiana un mismo contexto (hacer la colada, conducir un vehículo), el sujeto memoriza una representación estable, única, un esquema que se active siempre igual, en vez de construir una nueva representación situacional e inestable para cada contexto (Pozo, 1989).

Esta noción de esquema tiene sus precedentes en la obra de Piaget (1969) el cual lo define como un conjunto de unas estructuras complejas de datos que representan los conceptos genéricos almacenados de personajes, objetos y metas. Estos esquemas se construyen gracias a la capacidad inductiva especializada del ser humano, en apresar regularidades de su entorno (Rodrigo \& Correa, 2004). Según Pozo (2006), este es el primer proceso asociativo que permite vivir en un mundo más predecible y controlado, gracias a que al extraer regularidades se establecen secuencias predictivas de sucesos y conductas.

Lo importante no es el hecho de qué es lo que ocurre en realidad en el cerebro, sino cómo se representan los diferentes acontecimientos del jugador de baloncesto. Y estas representaciones esquemáticas están relacionadas con la formación de conceptos abstractos aunque, por lo que parece, éstos no están almacenados como tales, sino que dependiendo de la demanda concreta, se activarán partes de este concepto. Así, la activación que tiene un jugador de su esquema sobre un $\mathrm{BD}$ será diferente si participa como jugador que realiza el bloqueo, como jugador con balón, o como jugador que no interviene directamente. El bloqueador activará aquellas pautas relacionadas con cómo 
dificultar al adversario directo del compañero, para que no pase el bloqueo o en cómo orientar su cuerpo para la continuación. En cambio, el jugador con balón activará aquellas otras pautas relacionadas con cómo separase de su oponente, cuando pase el bloqueo, etc. Pero los jugadores que no participen en dicho bloqueo, también activarán un concepto diferente de $\mathrm{BD}$, recuperando las posibles trayectorias de sus compañeros, y los posibles pases que se pueden dar, etc.

Todo este proceso lo explica Pozo (1992) diciendo que el aprendiz relaciona unos hechos con otros, para así reconocer clases de objetos, a los que se les puede atribuir características similares, más allá de los cambios que se puedan producir de un hecho a otro. Con este proceso el jugador adquiere conceptos, aunque éstos, ya sean modelos contextuales o esquemas más genéricos, tiene una función descriptiva o predicativa, pero no explicativa. Sirven para predecir sucesos, pero no para comprenderlos (Pozo, 2006). En el caso anterior el jugador no sabe porqué tiene que dirigirse hacia el aro en el BD, si su adversario se coloca detrás de él. Para comprender esto, hay que hacer algo más.

Por este mismo proceso se forman, mediante un aprendizaje inconsciente, las teorías implícitas del jugador. Éstas "están elaboradas mediante esquemas de conocimiento que permite dar sentido al mundo que nos rodea" (Pozo, 2006, p. 92). Es decir que la consciencia no está directamente relacionada con la formación de esquemas de conocimiento. Esto quiere decir que se pueden formar conceptos abstractos de manera inconsciente. Es lo que ha sucedido con demasiada asiduidad en los deportes colectivos. El jugador ha construido esquemas sobre cómo se debe jugar, de manera inconsciente, basada en su experiencia repetida de éxitos y fracasos. Esto ha hecho que se generen conceptos equivocados de juego basados tanto en la no formación de ciertos conceptos, como en la falta de relación y aplicación de otros, en determinadas circunstancias de juego. Así, por ejemplo en baloncesto, es difícil que un jugador por sí solo aprenda que el concepto de profundizar en el espacio puede estar relacionado con un BI, y cuando pueda profundizar no lo haga, ya que el concepto de BI y de profundizar en el juego para él, no están relacionados. Esto es así puesto que el tipo de relaciones que se forman en las teorías implícitas (o de manera consciente con un aprendizaje asociativo), solo forman esquemas aislados de la realidad, sin conexión entre ellos. Pero, para la comprensión de un ámbito de conocimiento como cualquier deporte de equipo, no es suficiente relacionar unos hechos con otros (si sucede esto hay que hacer lo otro), sino que es necesario que se produzcan representaciones más elaboradas que las anteriores (Marmolejo-Ramos, 2007) en las que existan relaciones entre esos esquemas generales, es decir, entre conceptos (si sucede esto hay que hacer lo otro porque...). En el ejemplo anterior, hay que relacionar el concepto de BI y el de buscar profundizar cuando el adversario lo permita. Esto es así, porque sería imposible memorizar todas las posibles relaciones de los sucesos que se pueden dar en los deportes de equipo, al ser éstos prácticamente irrepetibles.

\section{- Según el grado de relación entre esquemas}

Para dotar de significado a un concepto, es decir, comprenderlo, es necesario que se dé un proceso de compresión gradual. No se puede comprender algo de inmediato y pretender tener el mismo conocimiento que una persona experta. Cada ensayo o intento va a proporcionar una nueva comprensión de lo que se quiere conocer, al ir relacionándose el concepto cada vez más y mejor con los que ya posee el jugador. Es decir, cuanto mayor sea el grado de relación entre esquemas, mayor el grado de comprensión. Esta idea parte de la teoría de Ausubel (1976), según la cual el sujeto puede adquirir conocimientos de una manera más o menos significativa, dentro de un continuo que va desde un aprendizaje memorístico hasta un aprendizaje significativo. Este grado de significatividad va a depender de cómo el sujeto haga suyo el nuevo aprendizaje, es decir, de cómo relacione el nuevo conocimiento con los que ya poseía. A medida que exista una mayor conexión con un número mayor de conceptos previos, más significativo será el aprendizaje conseguido. 
Según Pozo (1992) esta conexión de conceptos da como resultado principios. Éstos son conceptos muy generales y de un gran nivel de abstracción, y que suelen subyacer a la organización conceptual de un área, como es el caso de los deportes de equipo. Entre principios y conceptos más específicos, se forma una red de conceptos o jerarquía. Así, dentro de un área de conocimiento, un concepto está relacionado con otros conceptos, de forma que su significado proviene en gran medida de su relación con esos otros conceptos (Pozo, 1992). Kirk y Mcdonald (1998) señalan que la adquisición de habilidades en los deportes de equipo, conlleva una toma de decisiones apropiada y ésta no se podría producir, sin un cierto grado de comprensión, gracias a un aprendizaje activo del jugador.

Ausubel, Novak y Hanesian (1983) postulan que la estructura cognitiva del sujeto responde a una organización jerárquica, en la que los conceptos se conectan entre sí, mediante relaciones de subordinación, de los más generales a los más específicos. Por ejemplo, será más significativo el aprendizaje en baloncesto del concepto de profundizar en el juego, a medida que lo relacione con otros de niveles superiores (éste está incluido dentro del concepto de lanzar de manera eficiente) como del mismo nivel (para lanzar de manera eficiente también tengo que conseguir el menor grado de oposición).

Pero, para que se produzca el aprendizaje significativo, es necesario que se den tres condiciones: la primera, que el material que debe aprender el sujeto sea potencialmente significativo, desde un punto de vista lógico, es decir que posea una estructura y organización interna. Esto es lo que ocurre con los deportes de equipo, donde la actuación de sus participantes está regida por una lógica interna, formada por "reglas de acción" (action rules) o principios de juego que determinan el patrón de interacción entre los participantes (Grehaigne, Godbout \& Bouthier, 1999). La segunda condición es que el sujeto debe contar con unos conocimientos previos, que pueda relacionar con lo nuevo que se tiene que aprender. Por último, es necesario que el aprendiz quiera aprender de modo significativo. Para esta última condición, es muy importante la motivación que posea el jugador hacia este deporte, siendo la actuación del entrenador fundamental para que no se pierda.

Según Novak (1988), para que se produzca este aprendizaje significativo, se tiene que vincular la nueva información con conceptos ya existentes en la estructura cognitiva del que aprende, aunque esto no es del todo cierto, ya que, como se ha dicho, para que la nueva información se vincule con los conceptos ya existentes, ésta debe ser comprendida, es decir, el esquema estable al que alude la nueva información (ya sea construido de manera consciente o inconsciente) debe ser relacionada conscientemente con los conceptos previos. A este proceso, Novak (1998) lo llama aprendizaje de proposiciones, y lo define como la unidad principal de significado, donde un concepto se relaciona con otro. Así, la riqueza del significado de un concepto aumenta con el número de proposiciones que se aprenden de él, es decir, con el número de relaciones que se establezca con otros conceptos.

Si el jugador sólo vincula la nueva información (un modelo mental episódico de un BD) con los conocimientos ya existentes (un esquema general de $\mathrm{BD}$ ) pueden pasar dos cosas, que esta nueva información se asemeje a la que existía y se consolide el esquema de conocimiento (esto es lo que Pozo (2006) llama construcción estática de conocimiento), o si lo que está intentando aprender no coincide con los conceptos que el jugador posee, la nueva información se almacene de manera asilada, como un modelo episódico con pocas probabilidades de ser activado de nuevo, puesto que son las pautas de activación que se repiten más veces, es decir un esquema estable, las que tendrán conexiones más intensas y las que más se utilicen (Anderson, 1983; Rodrigo \& Correa, 2004). Esto es lo que ocurre cuando se utiliza un aprendizaje poco significativo, en el que el jugador no comprende el porqué de lo que tiene que hacer, porque no lo relaciona con lo que ya sabe. Por ejemplo, cuando un entrenador intenta enseñarle a un jugador con balón que durante un $\mathrm{BD}$, si su defensor se separa para pasar el bloqueo entre el bloqueador y el aro, debe pararse a tirar, pero si el jugador tiene implícito el concepto ir hacia el aro como única forma de aprovechar 
el bloqueo, será muy difícil que pueda cambiar su idea inicial. Escaño y Gil de la Serna (1992) y Pozo (1992) expresan que estas representaciones se caracterizan por su resistencia a cambiar, puesto que han sido adquiridas en el transcurso de sus experiencias durante muchos entrenamientos, y el jugador en esta nueva situación no detecta ningún conflicto que justifique modificar sus conocimientos previos, ya que no percibe ninguna falta de ajuste a la "realidad".

Por lo tanto, para que el jugador pueda adquirir el nuevo conocimiento de manera significativa (que el jugador se pare y tire tras un $\mathrm{BD}$, si su adversario se separa) debe comprender la nueva información, relacionando el concepto al que alude esta información (el BD) con conceptos que ya poseía (separarse del adversario para lanzar cuando exista oposición). Como dice Novak (1998): "el aprendizaje significativo implica relacionar nuevos conceptos mediante proposiciones válidas para la vida de la persona" (p.61).

Estos conceptos previos que van a ser cambiados son llamados por Ausubel (1976), inclusores, y sirven de base al nuevo conocimiento. Éstos son transformados produciéndose un nuevo significado fruto de la interacción entre ambos. La incorporación del nuevo contenido al inclusor hace que éste se desarrolle y amplíe (Martín \& Solé, 2004). Solo así se comprende y solo así se adquieren nuevos significados. "Aprender significados es cambiar mis ideas como consecuencia de su interacción con la nueva información” (Pozo, 1992, p. 35). Es lo que, más adelante, Pozo (2006) llamará "construcción dinámica del conocimiento".

Según el grado de cambio que se produzca en el concepto inclusor, se podrá hablar de diferentes procesos. Si el cambio que se produce, no modifica el esquema estable del inclusor y solo lo enriquece, modificando la organización jerárquica conceptual del jugador que se está produciendo, según Pozo (1989), "un ajuste", mientras que si en el cambio, el esquema de conocimiento inclusor es modificado, entonces se produce un proceso de "reestructuración". En ambos, se debe activar previamente el concepto inclusor mediante una reflexión, que implicará tomar conciencia de que los conceptos forman parte de un sistema de relaciones y no son entidades aisladas (Pozo, 1989). El proceso de ajuste, según Ausubel (1976), puede tener diferentes fases según cómo afecte la nueva información al orden jerárquico establecido por el jugador en su memoria. Por un lado, están los aprendizajes supraordinarios, aquellos en los que se aprende un concepto que abarca varias ideas ya presentes $\mathrm{o}$, al contrario, aprendizajes subordinados, es decir, cuando se aprende un concepto del que dependen otros ya conocidos. Por otro lado están los aprendizajes combinatorios, en los que se aprenden nuevos conceptos del mismo nivel en la jerarquía (Coll, 2004; Roman \& Diez, 1990). Cuando un jugador aprende el principio de evitar la aparición de ayudas, y comprende que abarca ideas que ya tenía, como realizar otras acciones de distracción simultaneas a la que está produciendo ventaja, tiene lugar un aprendizaje supraordinario (Figura 1). Cuando el jugador relaciona este concepto con otro superior en la jerarquía (dificultar la acción defensiva), el aprendizaje está siendo subordinado. Por último, cuando el jugador lo relaciona con otros conceptos que están en el mismo nivel, y que ya posee, el aprendizaje está siendo combinatorio; en este caso, cuando el jugador relaciona que este principio busca el mismo objetivo que el de dificultar la recuperación defensiva (Figura 1).

El segundo proceso por el cual se cambian los conocimientos previos, es el de reestructuración. Este cambio afecta al esquema estable de conocimiento que el jugador tiene de un concepto, y se produce cuando éste no da respuesta a nuevas situaciones que pueden acontecer en la realidad, y que se hacen frecuentes. Cuando sucede esto, lo primero que realiza el jugador es un ajuste a sus representaciones, pero cuando aún así esto sigue sin dar respuesta a lo que sucede, el jugador se ve obligado a realizar un cambio conceptual. Como dice Pozo (1989), para que se produzca una reestructuración, se requiere haber pasado antes por otras formas más elementales de aprendizaje como el ajuste, y que por un efecto acumulativo acaban por hacer necesario ese cambio conceptual. Cuando se dan estas condiciones en el entorno (o se generan), para poder cambiar la representación 
FIGURA 1

Ejemplo de jerarquización de principios de juego.

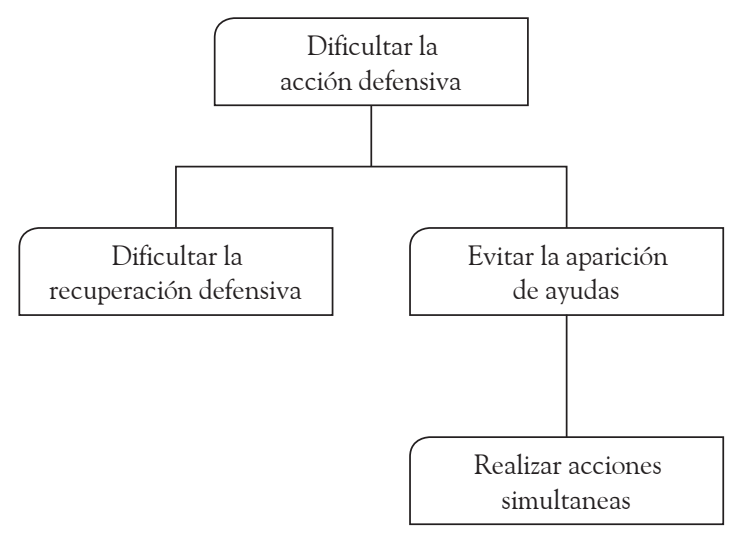

Fuente: elaboración propia.

errónea, primero hay que activarlas. Esto se consigue haciéndolas conscientes gracias a un proceso de reflexión (Coll, 1988; Coll et al., 2002; Pozo, 2006). El uso eficaz por parte del entrenador de una comunicación interrogativa que provoque la reflexión, permite a los jugadores construir en el cerebro redes semánticas más complejas, relacionándolas con la información que ya poseía. Este proceso se conoce como chunking (Tenenbaum, 2003). Una vez conseguido esto, hay que generar en el jugador un conflicto entre los conocimientos previos y los presentados como nuevos y válidos. Esto va a producir un desequilibrio en sus esquemas que hará sentir insuficientes estos conocimientos, lo que intentará equilibrar modificando los esquemas para adaptarse a las nuevas situaciones que se producen en el medio. Por lo tanto, los conflictos tienen un papel positivo en la adquisición de nuevos conocimientos (Inhelder, Sinclair \& Bovet, 1975). La corriente de enseñanza comprensiva de los deportes colectivos iniciada por Almond (1983) y Bunker y Thorpe (1982), hace suya esta idea para conseguir aprendizajes con significado. Para ello, los aprendices deben involucrarse activamente en el aprendizaje, tratando de relacionar lo que aprenden con lo que ya saben, siendo "constructores" de sus aprendizajes y quedando la función del entrenador como mediador entre el nuevo conoci- miento y el aprendiz (Metzler, 2000; Rink, 2001). Autores como Grehaigne et al. (1999) proponen que, para conseguirlo, las actividades deben mantener el marco contextual y problemático que se da en el juego real. Varios trabajos ya han señalado la relación entre el constructivismo y aprendizaje que busca conseguir el modelo comprensivo, como son los llevados a cabo por Grehaigne y Godbout (1995), Kirk y Macdonald (1998), Kirk y MacPhail (2002) o por Rink (2001).

\section{Atendiendo a los procesos de recuperación}

El proceso de recuperación de lo aprendido es algo complejo y dinámico, ya que al generarse nuevas conexiones, cada vez que se aprende algo, lo que se quiere recuperar ha adoptado otra organización diferente, con lo cual, a la hora de recuperar esa información, puede que no se logre o que aparezcan otras distintas en su lugar. Pero además, la recuperación se ve afectada por la forma en que se adquiere la información que se intenta recuperar (Pozo, 2006); así, a medida que existan un número mayor de relaciones entre esquemas, más fácil será la recuperación.

La atención y la conciencia están íntimamente ligadas. La atención puede tomar una dimensión externa, centrándose en los estímulos del entorno, o una dimensión interna, centrándose en los procesos internos de la memoria de trabajo. Pues a medida que el sujeto tenga una atención dirigida al exterior, será más inconsciente de esos procesos internos que se den en la memoria de trabajo, como la activación o recuperación de lo aprendido para ser comparado con la nueva información. Esto es lo que ocurre cuando el jugador automatiza ciertas acciones motrices. A medida que estas representaciones se adquieren condensadas, y se repiten en la práctica, se consigue que se automaticen, es decir, que se activen juntas con un consumo menor de recursos atencionales. Con la práctica repetida, estos aprendizajes se condensan hasta el punto de que pasan de ser actividades controladas en la memoria de trabajo, a ser rutinas automatizadas, sin apenas control consciente, siendo este proceso, 
específico de los conocimientos procedimentales (Pozo, 2006). Como anota Buceta (1998), en un proceso de aprendizaje de habilidades deportivas, en una primera fase, se requiere que el deportista de manera consciente atienda y discrimine los estímulos antecedentes y las respuestas propias relevantes, y dé las "ordenes" oportunas para ejecutar la nueva conducta. Posteriormente, existe una fase donde el deportista debe consolidar la nueva habilidad que ahora domina, de forma consciente para utilizarla de manera automática.

Según Anderson (1983), inicialmente, todo el conocimiento es consciente porque se hace declarativo, siendo la prueba de ello que se puede verbalizar. Con la práctica, este conocimiento se reemplaza por procedimientos que permiten desarrollar comportamientos de forma directa, inconscientemente. El sistema de producción representa procedimientos de tareas específicas en la memoria a largo plazo, que está activada sin la necesidad de tener conocimiento sobre los procedimientos por recuperar en la memoria de trabajo.

Aunque Pozo (2006) declara inicialmente que este proceso de automatización, al igual que el de condensación, pertenece a un aprendizaje asociativo, es decir que no existe comprensión por parte del sujeto de lo que hace, posteriormente, y como se ha indicado, afirma que la condensación, y la activación de esa agrupación, puede enriquecerse mediante procesos de comprensión. Por lo tanto, la automatización, al igual que la condensación de información, puede producirse mediante un proceso constructivo de aprendizaje. Algunos autores (Grehaigne, Godbout \& Bouthier, 2001; Musch et al., 2002) utilizan, en sus propuestas metodológicas, situaciones para la mejora de la capacidad de ejecución de los participantes, gracias a tareas que contengan elementos característicos de las prácticas deportivas estandarizadas, consiguiendo una automatización más comprensiva.

Una de las ventajas de esta condensación y automatización cuando es aprendida mediante procesos comprensivos, es que, aunque se active el procedimiento como un todo, el jugador, al comprender las relaciones con otras representaciones, si durante la realización del procedimiento surgiera algún cambio, sería capaz de modificar su conducta, adaptándose a las nuevas circunstancias de juego, cosa imposible si no hubiera existido un aprendizaje comprensivo. La diferencia, entre este aprendizaje y otro meramente asociativo, es que "cuando la tarea se complica o desvía de las previsiones, cuando deja de ser un ejercicio para convertirse en un problema, se puede recuperar el control de la misma" (Ericsson \& Smith, 1991, p. 145). Es lo que ocurre cuando se aprende a conducir un automóvil que, aunque se convierta en un proceso automático, cuando surge un imprevisto, el conductor recupera rápidamente el control de la tarea, concentrando sus recursos en los cambios.

En definitiva, es necesario entender que el aprendizaje de las habilidades requeridas para la práctica de los deportes de equipo, se debe realizar gracias a un proceso constructivo del jugador, en el que comprenda la naturaleza, las características y los objetivos de la actividad deportiva que practica, para que tenga una cierta idea acerca de el qué, el para qué, el por qué y el cuándo de su conducta, lo que le va a permitir dar sentido, significado y funcionalidad al cómo de sus acciones (Metzler, 2000; Grehaigne \& Godbout, 1995).

\section{Referencias}

Alarcón, F. (2008). Incidencia de un programa de entrenamiento para la mejora de la táctica colectiva del ataque posicional de un equipo de baloncesto masculino. Tesis doctoral no publicada, Universidad de Granada, España.

Almond, L. (1983). Games making. Bulletin of Physical Education, 19 (1), 32-35.

Anderson, J. R. (1976). Language, memory and thought. Hillsdale NJ: Erlbaum.

Anderson, J. R. (1982). Acquisition of cognitive skill. Psychological Review, 89, 369-406.

Anderson, J. R. (1983). The architecture of cognition. Cambridge: Havard University Press.

Anderson, J. R. (1987). Skill acquisition: Compilation of weak-method problem solutions. Psychological Review, 94, 192-210.

Aparicio-Serrano, A. \& Hoyos de los Rios, O. L. (2008). Enseñanza para el cambio de las representaciones 
sobre el aprendizaje. Universitas Psychologica, 7 (3), 725-737.

Ausubel, D. P. (1976). In defense of advance organizers: A reply to my critics. Review of Education Research, 48, 251-257.

Ausubel, D. P., Novak, J. D. \& Hanesian, H. (1983). Psicología Educativa: un punto de vista cognitivo. Mexico: Trillas.

Baddeley, A. (1990). Human memory. Theory and practice. Hillsdale, N.J.: Erlbaum.

Beltrán, J., García-Alcaniz, E., Moraleda, M., Calleja, G. \& Santiuste, V. (1992). Psicología de la Educación. Salamanca: Eudema.

Bunker, D. \& Thorpe, R. (1982). A model for the teaching of games in secondary schools. Bulletin of Physical Education, 18, 7-10.

Buceta, J. M. (1998). Psicología del entrenamiento deportivo. Madrid: Dykinson.

Carretero, M. (1985). Aprendizaje y desarrollo cognitivo: un ejemplo del tratado del inútil combate. En J. Mayor (Ed.), Actividad humana y procesos cognitivos (pp. 145-160). Madrid: Alhambra.

Chi, M.T.H. \& Rees, E. T. (1983). A learning framework for development: The control and construction of hierarchies of skill. Psychological Review, 87, 447-531.

Coll, C. (1988). Significado y sentido en el aprendizaje escolar. Reflexiones en torno al concepto de aprendizaje significativo. Infancia y Aprendizaje, 41, 131-142.

Coll, C. (1991). Concepción constructivista y planteamiento curricular. Revista Cuadernos de Pedagogía, 188, 8-11.

Coll, C. (1996). Psicología de la instrucció. Universitat Oberta de Catalunya. Barcelona: Laia.

Coll, C. (2004). Constructivismo y educación: la concepción constructivista de la enseñanza y el aprendizaje. En C. Coll, J. Palacios \& A. Marchesi (Eds.), Desarrollo psicológico y educación. Psicología de la educación escolar (Vol. 2, pp. 157-188). Madrid: Alianza Editorial.

Coll, C., Martí, E., Mauri, T., Miras, M., Onrubia, J., Solé, I. \& Zabala, A. (2002). El constructivismo en el aula. Barcelona: Grao.

Coll, C. \& Valls, E. (1992). El aprendizaje y la enseñanza de los procedimientos. En C. Coll, J. I. Pozo, B.
Sarabia \& E. Valls, Los contenidos en la reforma (pp. 81-132). Madrid: Santillana.

De Vega, M., Díaz, J. \& León, I. (1999). Procesamiento del discurso. En M. De Vega \& F. Cuetos (Eds), Psicolingüística del español (pp. 271-306). Madrid: Trotta.

Ericsson, K. A. \& Smith, J. (1991). Toward a general theory of expertise. Prospects and limits. Cambridge, Mass: Cambridge University Press.

Escaño, J. \& Gil de la Serna, M. (1992). Cómo se aprende y cómo se enseña. Barcelona: ICE/UB Horsori.

French, K. E. \& Thomas, J. R. (1987). The relation of knowledge development to children's basketball performance. Journal of Sport Psychology, 9, 15-32.

García, J. E. (1995). Epistemología de la complejidad y enseñanza de la ecología. Tesis Doctoral, Facultad de las ciencias de la Educación, Universidad de Sevilla, España.

Grehaigne, J. F. \& Godbout, P. (1995). Tactical knowledge in team sports from a constructivist and cognitivist perspective. Quest, 47 (4), 490-505.

Grehaigne, J. F., Godbout, P. \& Bouthier, D. (1999). The foundations of tactics and strategy in team sports. Journal of Teaching in Physical Education, 18 (2), 159-174.

Grehaigne, J. F., Godbout, P. \& Bouthier, D. (2001). The teaching and learning of decision making in team sports. Quest, 53 (1), 59-76.

Inhelder, B., Sinclair, H. \& Bovet, M. (1975). Aprendizaje y estructuras del conocimiento. Madrid: Morata.

Kirk, D. \& Macdonald, D. (1998). Situated learning in physical education. Journal of Teaching in Physical Education, 17 (3), 376-387.

Kirk, D. \& MacPhail, A. (2002). Teaching games for understanding and situated learning: Re-thinking the Bunker-Thorpe model. Journal of Teaching in Physical Education, 21 (2), 177-192.

Marmolejo-Ramos, F. (2007). Nuevos avances en el estudio científico de la comprensión de textos. Universitas Psychologica, 6 (2), 331-343.

Martí, E. (1995). Metacognición: entre la fascinación y el desencanto. Infancia y Aprendizaje, 72, 9-32.

Martín, E. \& Solé, I. (2004). El aprendizaje significativo y la teoría de la asimilación. En C. Coll, J. Palacios \& A. Marchesi (Coords.), Desarrollo psicológico y 
educación. Psicología de la educación escolar (Vol. 2, pp. 89-116). Madrid: Alianza Editorial.

Metzler, M. (2000). Instructional models for physical education. Boston: Allyn and Bacon.

Musch, E., Mertens, B., Graça, A., Timmers, E., Meertens, T., Taborsky, F. et al. (2002, July). An innovated didactical invasion games model to teach basketball and handball [CD]. The 7th Annual Congress of European College of Sport Science, Athens, Greece.

Novak, J. D. (1988). Aprendiendo a aprender. Barcelona: Martínez Roca.

Núñez, J. P. (1998). Aprendizaje inconsciente. Condicionamiento a estímulos visuales subliminales. Tesis doctoral no publicada, Universidad Pontificia de Madrid, España.

Piaget, J. (1969). Psicología y pedagogía. Barcelona: Crítica.

Pozo, J. I. (1989). Teorías cognitivas del aprendizaje. Madrid: Visor.

Pozo, J. I. (1992). El aprendizaje y la enseñanza de hechos y conceptos. En C. Coll , J. I. Pozo, B. Sarabia \& E. Valls (Eds.), Los contenidos en la reforma (pp. 19-80). Buenos Aires: Santillana.

Pozo, J. I. (2002). La adquisición de un proceso científico como cambio representacional. Investigações em Ensino de Cięncias, 7 (3), 1-30.

Pozo, J. I. (2006). Aprendices y Maestros. Madrid: Alianza Editorial.
Reber, A. S. (1993). Implicit learning and tacit knowledge. New York: Oxford University Press.

Rink, J. E. (2001). Investigating the assumptions of pedagogy. Journal of Teaching in Physical Education, 20 (2), 112-128.

Roman, M. \& Diez, E. (1990). Currículum y Aprendizaje. Madrid: Itaka.

Rodrigo, M. J. (1994). Contexto y desarrollo social. Madrid: Síntesis.

Rodrigo, M. J. \& Correa, N. (2004). Representación y procesos cognitivos: esquemas y modelos mentales. En C. Coll, J. Palacios \& A. Marchesi (Eds.), Desarrollo psicológico y educación. Psicología de la educación escolar (Vol.2, pp. 117-136). Madrid: Alianza Editorial.

Ruiz, L. M. \& Arruza, J. (2005). El proceso de toma de decisiones en el deporte. Clave de la eficiencia y el rendimiento óptimo. Barcelona: Paidós.

Santiuste-Bermejo, V. \& Santiuste-Díaz, M. (2008). Consistencia epistémica del síndrome de dificultades de aprendizaje: aportaciones de la magnetoencefalografía como técnica de neuroimagen funcional. Universitas Psychologica, 7 (3), 655-671.

Tenenbaum, G. (2003). Expert athletes: An integrated approach to decision making. En J. L. Starkets \& K. A. Ericsson (Eds.), Experts performance in sports: Advances in research on sport expertise (pp. 191. 218). Champaign, IL: Human Kinetics. 